\title{
Self administered medical abortion pills: evaluation of the clinical outcome and complications among women presenting with unsupervised pill intake to a tertiary care hospital in Malwa region of Punjab, India.
}

\author{
Shivali Bhalla ${ }^{1 *}$, Lajya Devi Goyal ${ }^{1}$, Shivam Bhalla ${ }^{2}$, Balpreet Kaur ${ }^{1}$
}

\begin{abstract}
${ }^{1}$ Department of Obstetrics and Gynecology, Guru Gobind Singh Medical College and Hospital, Faridkot, Punjab, India ${ }^{2}$ Department of Surgery, Government Medical College and Hospital, Patiala, Punjab, India
\end{abstract}

Received: 31 January 2018

Accepted: 06 March 2018

\section{*Correspondence:}

Dr. Shivali Bhalla,

E-mail: manasmansha@gmail.com.

Copyright: (C) the author(s), publisher and licensee Medip Academy. This is an open-access article distributed under the terms of the Creative Commons Attribution Non-Commercial License, which permits unrestricted non-commercial use, distribution, and reproduction in any medium, provided the original work is properly cited.

\section{ABSTRACT}

Background: Unsupervised medical abortion pill intake is becoming a public health hazard. Because of its widespread misuse, we planned to undertake this study to evaluate the clinical presentation and outcome of such patients.

Methods: Hundred patients meeting inclusion criteria were included. Clinical presentation, treatment given and, outcome and complications were analyzed.

Results: Majority (53\%) patients were aged between 30-39 years. Seventy-seven percent were gravida three or more. Only $26 \%$ had taken the pill within prescribed gestational age limit for MTP (<seven weeks). Mid-trimester pill intake was encountered in $13 \%$ patients. Pill was consumed without any prior medical consultation by $73 \%$ patients. Majority (49\%) presented with incomplete abortion requiring surgical evacuation. Anaemia was most common associated co-morbidity in $80 \%$ patients and $28 \%$ required blood transfusions. Twenty-four percent patients presented with life threatening shock. Sepsis was noted in 3\% patients. Emergency laprotomy was required in $11 \%$ cases. Continuation of pregnancy was reported in $13 \%$ patients. Unintended pregnancy and failure of contraception were main reasons cited for abortion by $38 \%$ and $26 \%$ women. Non-contraceptive users constituted $48 \%$ patients . Over the counter easy availability was the main reason for unsupervised pill intake in $58 \%$ cases.

Conclusions: Unauthorized over the counter pill availability despite legal ban and ignorance of women have led to increased number of unsafe abortions. Strict legislations need to be imposed. Increasing awareness among women regarding complications of unsupervised pill intake and easy availability of safe contraceptive methods can help control this problem.

Keywords: MTP pill, Over the counter, Self-medication, Unsafe abortion, Unwanted pregnancy

\section{INTRODUCTION}

Unwanted pregnancy is common problem globally. According to World Health Organization, 19 million women worldwide undergo unsafe abortion annually and
18.5 million of these cases occur in developing nations. Mortality attributed to unsafe abortion is about 68,000 per year. In India, around 6.4 million abortions occur annually and among these $56 \%$ are unsafe which accounts for $8-20 \%$ of all maternal deaths. 
Medical abortion is safer alternative to surgical abortion as it avoids the complications like infection, uterine perforation, cervical trauma and cervical incompetence. But, it is a blessing, only if, used according to standard protocol and under proper medical guidance. In India it is becoming a public health problem due to easy over the counter availability despite legal ban, widespread misuse by non -allopath doctors, dais and quacks and ignorance on part of women. The pills are being dispensed blindly without proper medical evaluation and even without ruling out the contraindications.

The guidelines for medical abortion in India have been prepared by WHO in human reproduction, All India Institute of Medical Sciences, in collaboration with Ministry of Health and Family Welfare, Government of India and Indian Council of Medical Research according to which medical abortion is approved up to seven weeks of pregnancy. ${ }^{1}$

Acceptability of minimum three follow -up visits, understanding the instructions, ready for surgical procedure if failure or excessive bleeding occurs, family support and easy access to appropriate health care facility are the pre-requisites. After taking formal consent, the recommended protocol is oral mifipristone $200 \mathrm{mg}$ on day 1 followed by misoprostol $800 \mathrm{mcg}$ vaginally or 400 mcg misoprostol orally, after 36-48 hrs, given under medical supervision. As unsupervised intake of medical abortion pills is rampant despite strict national policies and is taking a high toll on health of reproductive age group women we planned to conduct this study. The objectives of our study were to evaluate the clinical presentation and outcome of these patients.

\section{METHODS}

It is a prospective study including 100 patients presenting to outpatient and emergency department of Obstetrics and Gynaecology, Guru Gobind Singh Medical College and Hospital, Faridkot, Punjab between August 2016 and August 2017 with history of unsupervised intake of Medical abortion pill .Unsupervised pill intake implies when the pill is procured over the counter without authorized medical prescription and/or the pill has been prescribed by untrained personnel like dais and quacks and /or consumed without following protocol as laid down by Medical Termination of Pregnancy Act of India (MTP Act). Approval of the ethical committee of the institution was taken.

Detailed history was collected from the patients regarding age, parity, education status, last menstrual period, presenting complaints, source of pill procurement, reason for abortion, time interval between pill intake and visit to hospital and reason for avoiding tertiary centre as first approach. The detailed examination and ultrasonography was done and according to the condition of the patient, treatment was given and the outcome and complications analyzed.

\section{RESULTS}

Demographic profile showed majority of the women (53\%) belonged to age group of 30-39 years. Most of the women $(77 \%)$ were illiterate or had formal education only of primary level. Majority $(87 \%)$ belonged to low socio-economic status.

Table 1: Demographic details of the patients $(n=100)$.

\begin{tabular}{|c|c|c|}
\hline Parameter studied & \multicolumn{2}{|c|}{$\%$ Patients } \\
\hline \multicolumn{3}{|l|}{ Age (years) } \\
\hline $20-29$ & \multicolumn{2}{|l|}{43} \\
\hline $30-39$ & \multicolumn{2}{|l|}{53} \\
\hline $40-49$ & \multicolumn{2}{|l|}{4} \\
\hline Education status & Patients & Spouse \\
\hline Nil & 26 & 22 \\
\hline Primary & 51 & 42 \\
\hline Secondary & 13 & 22 \\
\hline High school & 7 & 7 \\
\hline College & 3 & 7 \\
\hline \multicolumn{3}{|l|}{ Gravida } \\
\hline 1 & \multicolumn{2}{|l|}{3} \\
\hline 2 & \multicolumn{2}{|l|}{20} \\
\hline 3 & \multicolumn{2}{|l|}{60} \\
\hline 4 or $>4$ & \multicolumn{2}{|l|}{17} \\
\hline
\end{tabular}

Nearly $3 / 4$ of the women, $(77 \%)$ were gravid three or more (Table 1). Majority, $54 \%$ patients procured the pill from non-allopath doctors, dais or quacks. Pill was procured by husband or relative in $37 \%$ cases (Figure 1).

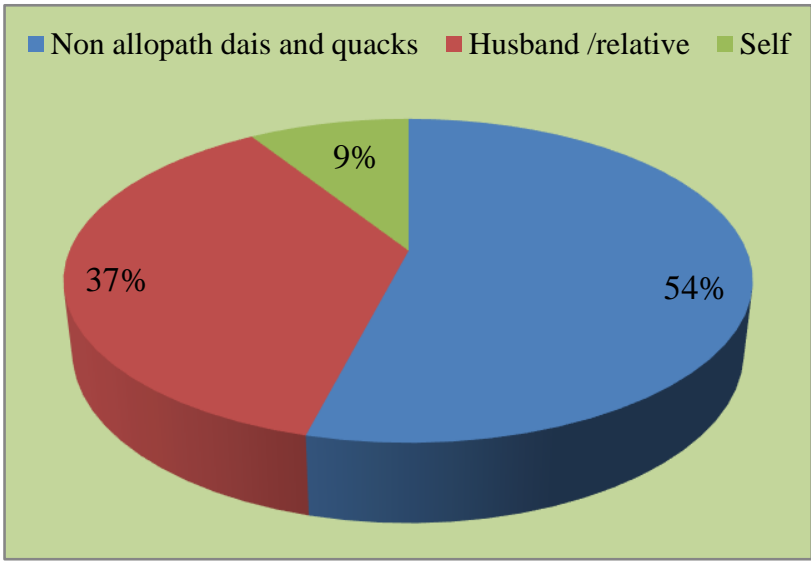

Figure 1: Source of pill procurement.

Only $26 \%$ of the women had taken the pill within the prescribed gestational age limit of up to seven weeks. Majority (61\%) had history of pill intake beyond seven weeks gestation but within first trimester \& $13 \%$ of the women had mid-trimester pill intake leading to increased complications. Majority of the patients $(69 \%)$ attended the hospital between 0-14 days of pill intake. Six percent women presented beyond one month. Majority of patients $(66 \%)$ had consumed the pill without prior ultrasonography to confirm the gestational age or localize 
the pregnancy. Unintended pregnancy, limiting family size and failure of contraception were the main cited reasons for abortion by $38 \%, 36 \%$ and $26 \%$ women respectively (Table 2).

Table 2: Gestational age at pill intake (from LMP), time interval $b / w$ intake of pill and visiting to hospital, reasons cited for abortion.

\begin{tabular}{|l|l|}
\hline Parameter studied & $\begin{array}{l}\% \\
\text { Patients }\end{array}$ \\
\hline Gestational age at pill intake (weeks) & \\
\hline$<7$ & 26 \\
\hline $7.1-10$ & 45 \\
\hline $10.1-13$ & 16 \\
\hline $13.1-16$ & 11 \\
\hline$>16$ & 2 \\
\hline Time interval (between pill intake at visit to \\
\hline hospital in days) & \\
\hline $0-7$ & 34 \\
\hline $8-14$ & 35 \\
\hline $15-21$ & 29 \\
\hline $22-28$ & 6 \\
\hline$>28$ & 6 \\
\hline Ultrasonography prior to pill intake & \\
\hline Yes & 34 \\
\hline No & 66 \\
\hline Reason cited for abortion & 38 \\
\hline Unintended pregnancy & 36 \\
\hline Limiting family size & 26 \\
\hline Failure of contraception & \\
\hline
\end{tabular}

Easy over the counter pill availability was cited by $58 \%$ patients as a quicker solution to their problem than to indulge into more complicated procedures at authorised medical centres.

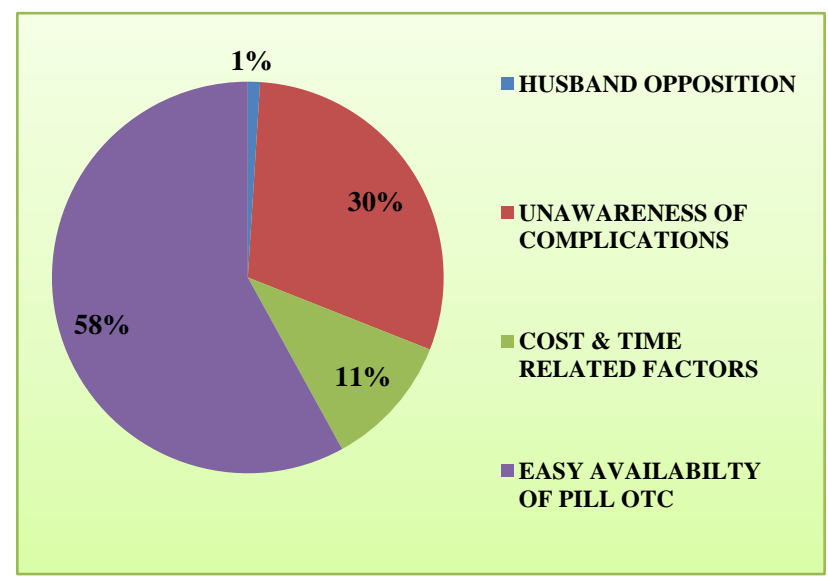

Figure 2: Reason cited for avoiding authorised medical centre as first approach.

Unawareness of the complications of unsupervised pill intake and time and cost related factors made $30 \%$ and $11 \%$ patients, respectively, to avoid these centres (Figure 2).
Majority (49\%) patients presented with bleeding per vaginum along with passage of fleshy mass. Prolonged irregular bleeding $\mathrm{P} / \mathrm{V}$ was encountered in $26 \%$ patients. Twenty-four percent patients presented with life threatening shock. Continuation of pregnancy to late first trimester and mid-trimester was reported by $13 \%$ women. Three percent women presented with sepsis (Table 3).

Table 3: Chief presenting complaints of the patients presenting with unsupervised intake of MTP pills.

\begin{tabular}{|lc|}
\hline $\begin{array}{l}\text { Chief complaints } \\
\text { Heavy bleeding with passage of fleshy }\end{array}$ & Patients \\
\hline Irregular bleeding per vaginum & 49 \\
\hline Shock & 26 \\
\hline Pain abdomen & 24 \\
\hline Continued pregnancy & 13 \\
\hline $\begin{array}{l}\text { Fever with pain abdomen and foul- } \\
\text { smelling discharge per vaginum }\end{array}$ & 13 \\
\hline
\end{tabular}

Majority (49\%) of the patients had incomplete abortion. Anaemia of varying degrees was the most common associated medical co-morbidity in $80 \%$ patients and $28 \%$ had severe anaemia necessitating blood transfusions. Nearly quarter of the patients $(24 \%)$ presented in state of shock. Complete abortion was seen only in $19 \%$ cases. In $13 \%$ cases pregnancy continued undisturbed. Ultrasonography revealed missed abortion and hydatiform mole in $7 \%$ and $3 \%$ cases. Ruptured ectopic pregnancy was diagnosed in $5 \%$ patients and $1 \%$ had scar pregnancy, thus requiring immediate laprotomy. Three percent presented with sepsis. No case of maternal mortality was reported in our study (Table 4).

Table 4: Outcome (clinical and USG) of unsupervised intake of MTP pills among study group.

\begin{tabular}{|l|l|}
\hline Outcome & $\%$ patients \\
\hline Incomplete abortion & 49 \\
\hline Shock & 24 \\
\hline Anaemia & 80 \\
\hline Mild to moderate anaemia & 52 \\
\hline Severe anaemia & 28 \\
\hline Complete abortion & 19 \\
\hline Continuation of pregnancy & 13 \\
\hline Missed abortion & 7 \\
\hline Ectopic pregnancy & 5 \\
\hline Hydatiform mole & 3 \\
\hline Sepsis & 3 \\
\hline Scar pregnancy & 1 \\
\hline Maternal death & 0 \\
\hline
\end{tabular}

Surgical curettage was performed in $53 \%$ of the patients. Blood transfusion was required in $28 \%$ patients and $52 \%$ were prescribed oral or intravenous iron therapy. Around a quarter of patients $(24 \%)$ presented in shock requiring urgent resuscitative management. Uterotonic agents to 
complete the abortion process was required in $20 \%$ patients. Emergency laprotomy was required in $11 \%$ and in 5\% hysterotomy was required as a life saving measure. Cases of sepsis were managed by vigorous antibiotics and other supportive management. (Table 5).

Table 5: Treatment received by the patients presenting with unsupervised intake of MTP pills at the tertiary care centre.

\begin{tabular}{|ll|}
\hline Treatment received & $\%$ Patients \\
\hline Medical & 49 \\
\hline Management of shock & 24 \\
\hline Intravenous oxytocin & 10 \\
\hline Misoprostol tablets & 10 \\
\hline Supportive (I.V. antibiotics) & 5 \\
\hline Suction and evacuation & 53 \\
\hline Blood transfusion & 28 \\
\hline Oral iron therapy & 49 \\
\hline Intravenous iron therapy & 3 \\
\hline Exploratory laparotomy & 11 \\
\hline Ectopic pregnancy & 5 \\
\hline Scar pregnancy & 1 \\
\hline Hysterotomy & 5 \\
\hline Treatment not required & 10 \\
\hline
\end{tabular}

Details of contraception method revealed $48 \%$ women as non-users and $27 \%$ being dependent on highly unreliable barrier methods prior to abortion.

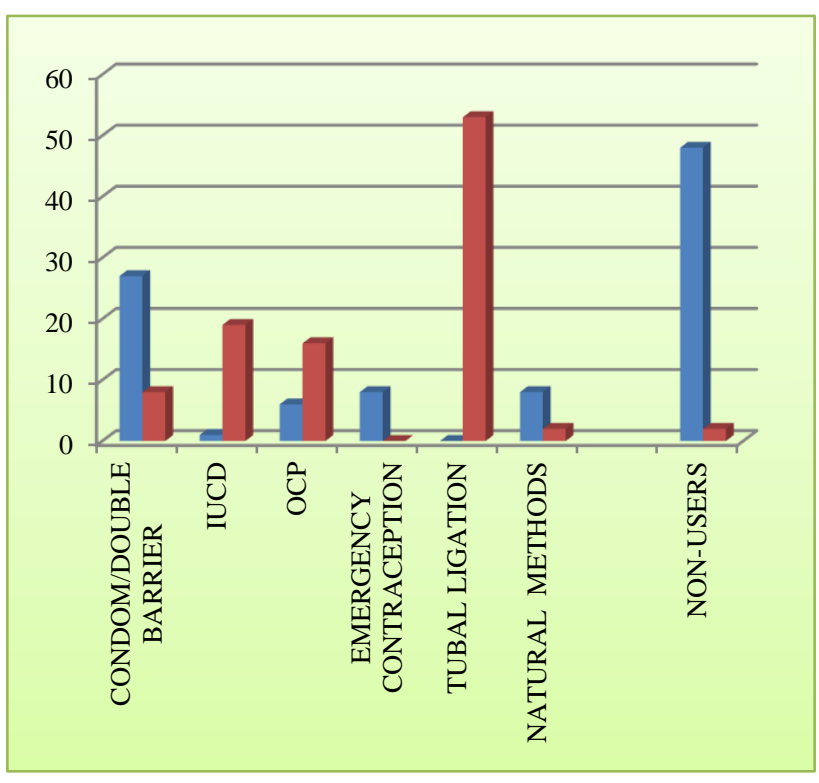

Figure 3: Preferred method of contraception used before and after abortion.

The use of OCP and IUCD was negligible among women prior to abortion but after proper counselling $16 \%$ and 19 $\%$ women agreed to use them. Fifty-three percent of the patients showed willingness for tubal ligation and $17 \%$ underwent ligation during treatment (Figure 3).

\section{DISCUSSION}

Unsafe abortion is an important cause of increased maternal morbidity and mortality in developing countries. The advent of medical abortion pill was intended to protect women from complicated surgical procedure of abortion. However, its widespread misuse by untrained personnels, ignorance and unawareness of complications of unsupervised intake on part of women and easy over the counter availability of the pill despite legal ban has made this pill a public health hazard. ${ }^{2}$

In our study, pill misuse was more widespread among low socio-economic strata women $(87 \%)$ owing to their ignorance and unawareness. Most of the women (77\%) and their spouses $(64 \%)$ in our study were either illiterate or had a formal education only of the primary level, hence, making them fall prey in hands of untrained and unauthorised pill providers. More than three-fourth of the women, $(77 \%)$ were gravida three or more. These results were seen to be similar to studies by Mishra et al (78\%), Giri et al (83\%), Thaker et al $(89.1 \%)$ and Kumari et al $(86.67 \%) .^{3-6}$

As per MTP Act of India, the safe upper gestational age limit for pill intake is upto seven weeks. The risk of complications associated with all forms of abortion increases with gestational age, rising exponentially after first trimester even if performed under the best circumstances. In our study, only a minority (26\%), had taken the pill at the recommended gestational age of upto seven weeks, which, is similar to a previous study conducted by Mishra et al (37\%). ${ }^{3}$ In present study, $61 \%$ of women had history of pill intake beyond seven weeks period of gestation but within first trimester \& $13 \%$ of the women presented with mid-trimester pill intake leading to increased complications.

Majority of the patients (69\%) attended the hospital between 0-14 days of pill intake, which is similar to a previous study by Agarwal $\mathrm{M}$ et al $(76.66 \%){ }^{7}$ Six percent women presented to our centre beyond one month of pill intake. Majority of patients had consumed the pill without any prior medical consultation (73\%). Unintended pregnancy, limiting family size and failure of contraception were the main cited reasons for abortion by $38 \%, 36 \%$ and $26 \%$ women respectively.

Majority $(67 \%)$ of the patients, procured the pill from non-allopath doctors, dais, quacks and chemists. These results are similar to studies by Agarwal $\mathrm{M}$ et al $(73 \%)^{(7)}$ and also by Kumari $\mathrm{R}$ et al $(70 \%){ }^{6}$ Hence this depicts the true scenario, that despite legal ban on over the counter supply of this drug and strict guidelines of MTP Act, the drug is being widely misused.The main reason cited by the patients for avoiding authorised medical centres as first approach to treatment was easy over the counter pill availablilty in $58 \%$ cases as it meant a quick solution to their problem than to indulge into more cumbersome procedures at authorised centres.. 
The most frequent complaint for admission was heavy vaginal bleeding along with history of passage of fleshy mass, suggesting incomplete abortion in $49 \%$ patients. This finding collaborates to other studies by Agarwal M et al, Nivedita et al, Thaker et al, Jethani $\mathrm{M}$ et al where reported rates of incomplete abortion were $56 \%, 62.5 \%$, $70.2 \%$ and $57.45 \%$ respectively. ${ }^{7-9}$ Surgical evacuation was required in $53 \%$ of the patients. This is similar to studies by Kumari $\mathrm{R}$ et al and by Mishra $\mathrm{N}$ et al where surgical curettage was required in $50 \%$ and $46.5 \%$ of the cases respectively. ${ }^{6,3}$ In medical abortion practice, method failure is said to occur when due to any reason a woman needs surgical evacuation to complete the abortion. Nearly $2-10 \%$ of women who otherwise choose supervised medical abortion would end up in surgical evacuation. But in our study this rate is very high which actually points out to method failure owing to erroneous pill prescription schedules by untrained personnel. This defeats the very purpose of medical abortifacient pill intake. Due to erratic drug schedules and /or incomplete regimens, $26 \%$ women in our study had complaints of prolonged irregular vaginal bleeding. In India where prevalence of anaemia among women is already high, such bleeding, further worsens the condition. Repeat administration of one or more doses of misoprostol or iv oxytocin to remove the retained bits of conception was required in $20 \%$ cases.

The most common associated medical co-morbidity was anaemia of varying degrees noted in $79 \%$ patients. The incidence of co existing anaemia was comparable to other studies by Agarwal $\mathrm{M}$ et al and Giri et al i.e. $79 \%$ and $76.66 \%$. $^{7,4}$ Twenty eight percent had severe anaemia, requiring blood transfusions. These results were similar to another study by Kumari R et al where $23.3 \%$ women had severe anaemia requiring blood transfusions. ${ }^{6}$ More than half $(52 \%)$, had mild to moderate anaemia which necessitated oral or intravenous iron therapy. Nearly a quarter of patients $(24 \%)$ presented with life threatening shock requiring urgent resuscitation, accounting for near miss mortality cases in our study. Ideally any method of medical abortion should have ongoing pregnancy rate of less than $1 \%$ but in our study continuation of pregnancy to late first trimester and mid-trimester was reported in $13 \%$ women. ${ }^{6}$ The high incidence of continued pregnancy strongly points out to erroneous and incomplete dosing schedules with which the drug is prescribed by the unauthorised personnel. Serious life-threatening complications like sepsis are common in women undergoing unsafe abortions. ${ }^{10,11}$ In our study, $3 \%$ women presented with features of sepsis like fever, pain abdomen etc. This is similar to the study by Agarwal $\mathrm{M}$ et al where sepsis rate was $3.33 \% .^{7}$

Ultrasonography should be done prior to prescribing the pill for pregnancy localization and confirmation of the gestational age. But our study revealed, it was not done in majority of patients (66\%). Ultrasonography done in our hospital revealed diagnosis of missed abortion, ruptured ectopic pregnancy and molar pregnancy in $7 \%, 5 \%$ and
$3 \%$ cases respectively. One case had scar pregnancy at site of previous caeserean scar thus requiring emergency hysterotomy.

In present study, $20 \%$ patients had history of one or more prior cesarean sections. This category is more vulnerable to life threatening complications as there is high risk of scar rupture .In one study by Potdar $\mathrm{J}$, the incidence of uterine rupture of a previous caeserean section scar during medical abortion in early pregnancy was 3 in $768 .{ }^{12}$ There is documented risk of $0.28 \%$ of uterine rupture in cases of mid trimester abortion with prior caeserean section delivery. ${ }^{13}$ In our study $5 \%$ of such cases required hysterotomy owing to risk of scar rupture.

Majority of the women were not aware of the contraceptive options available at health centres, hence, accounting for high (48\%) contraceptive non-users in our study, hence points to high unmet need of contraception. This scenario is alarming as the women are not using proper contraceptive methods despite the various national programmes propagating their use. This relavation in this study coincides with similar findings in another study by De Silva WI and others. ${ }^{14}$ Prior to abortion, $35 \%$ women were dependent on highly unreliable natural /barrier methods of contraception. The use of hormonal contraception and intrauterine contraceptive devices was negligible among women prior to abortion (6\% and $1 \%$ ). The patients were counselled during their treatment and were offered caffetaria approach for use of contraception. As a result, the use of IUCD and OCP increased to $19 \%$ and $16 \%$ post abortion. The use of condom dropped from $27 \%$ to $8 \%$. Fifty three percent patients who had completed their families showed willingness for tubal ligation and $17 \%$ underwent ligation during treatment at our hospital.

\section{CONCLUSION}

Medical abortion method should be properly used under strict supervision of trained professionals else it will continue to prove to be a health hazard. Strict legislations by national bodies needs to be imposed to curb this issue. Also, increasing awareness among women regarding complications of unsupervised pill intake and regarding availability and safety of various contraceptive methods can help control this hazard. Besides this, there is a need for strengthening the contraception use among women.

\section{ACKNOWLEDGMENTS}

Authors would like to thank Mandeep Kumar Tiwary for his support during study.

Funding: No funding sources Conflict of interest: None declared

Ethical approval: The study was approved by the Institutional Ethics Committee with GGSMCH, ethical approval No: GGS/IEC/17/32 


\section{REFERENCES}

1. Guidelines for medical abortion in India available at http://ebookbrowsee.net/guidelines-for-medicalabortion-in-india-doc-d134818684

2. Guidelines for medical abortion in India Material circulated by CASSA during the state level Workshop on "Review of MTP Act 1971 in the context of Women's Right to Safe Abortion and Halting Sex Selective Abortion, held in Chennai on 17th and 18th August 2007.

3. Mishra N. Unprecedented use of medical abortion can be injurious to health. JEMS. 2013;2(8):856-9.

4. Giri A, Srivastav VR, Suwal A, Sharma B. A study of complications following Self administration with Medical Abortion Pills. Nepal J Obstet Gynaecol. 2015;10(1):20-4.

5. Thaker RV, Deliwala KJ, Shah PT. Self medication of abortion pill: women's health in Jeopardy. NHLJMS. 2014;3(1):26-31.

6. Kumari R, Sharma A, Najam R, Singh S, Roy P. Mortality and morbidity associated with illegal use of abortion pill: a prospective study in tertiary care center. Int J Res Med Sci. 2016;4(7):2598-602.

7. Agarwal M, Datta A. How safe are over the counter abortion pills-differences between its intended and practical usage and its implications-a study conducted in a tertiary care centre in Shillong, Meghalaya, India. Int J Reprod Contracept Obstet Gynecol. 2016;5(9):3036-40.

8. Nivedita K, Fatima S. Is it safe to provide abortion pills over the counter? A study on outcome following self-medication with abortion pills. J Clin Diagn Res. 2015;9(1):1-4
9. Jethani M, Yadav K, Muchhoria S, Sharma S, Monika. Self Medicated Abortion-Care or Crime JMSCR. 2015;03(09):7507-12.

10. International Consensus Conference on Non-surgical (Medical) Abortion in Early First Trimester on Issues Related to Regimens and Service Delivery: frequently asked clinical questions about medical abortion. Bellagio: WHO;2006. Available at http://who.int/reproductivehealth/publications/medical_abortion/faq.pdf

11. Stillman M, Frost JJ, Singh S, Moore AM, Kalyanwala S. Abortion in India: a literature review. Guttmacher Institute. 2014;12-4.

12. Potdar J. Medical abortion with mifepristonemisoprostol in previous caesarean section, up to seven weeks gestation-a retrospective analysis of data. Indian Obstet Gynaecol. 2011;1(4):330-1.

13. Goyal V. Uterine rupture in second-trimester misoprostol-induced abortion after cesarean delivery: a systematic review. Obstet Gynecol. 2009;113(5):1117-23.

14. De Silva WI, Dayananda RA, Nishanthi Perera NW. Contraceptive behaviour of abortion seekers in Sri Lanka. Asian Popul Stud.2006;2(1):3-18.

Cite this article as: Bhalla S, Goyal LD, Bhalla S, Kaur B. Self administered medical abortion pills: evaluation of the clinical outcome and complications among women presenting with unsupervised pill intake to a tertiary care hospital in Malwa region of Punjab, India. Int J Reprod Contracept Obstet Gynecol 2018;7:1537-42. 